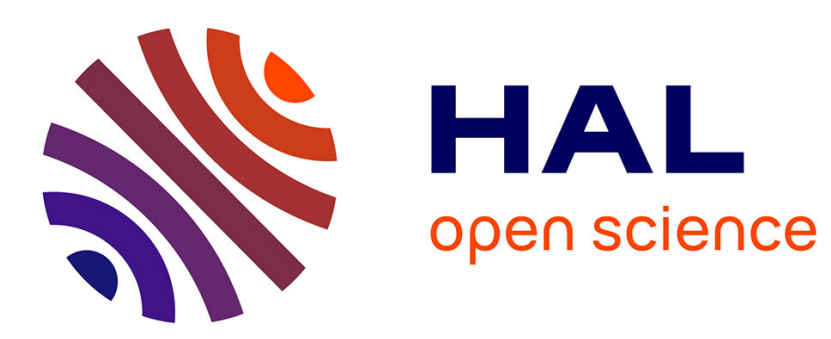

\title{
Oscillatory Kelvin-Helmholtz instability. Part 2. An experiment in fluids with a large viscosity contrast
}

Harunori Yoshikawa, José Eduardo Wesfreid

\section{To cite this version:}

Harunori Yoshikawa, José Eduardo Wesfreid. Oscillatory Kelvin-Helmholtz instability. Part 2. An experiment in fluids with a large viscosity contrast. Journal of Fluid Mechanics, 2011, 675, pp.249-267. 10.1017/S0022112011000152 . hal-00906513

\section{HAL Id: hal-00906513 \\ https://hal.science/hal-00906513}

Submitted on 19 Nov 2013

HAL is a multi-disciplinary open access archive for the deposit and dissemination of scientific research documents, whether they are published or not. The documents may come from teaching and research institutions in France or abroad, or from public or private research centers.
L'archive ouverte pluridisciplinaire HAL, est destinée au dépôt et à la diffusion de documents scientifiques de niveau recherche, publiés ou non, émanant des établissements d'enseignement et de recherche français ou étrangers, des laboratoires publics ou privés. 


\title{
Oscillatory Kelvin-Helmholtz instability: Part 2. An experiment in fluids with a large viscosity contrast
}

\author{
HARUNORI N. YOSHIKA WA $\dagger$ \\ andJ OSÉ EDUARDO WESFREID \\ Physique et Mécanique des Milieux Hétérogènes (PMMH) \\ UMR 7636 CNRS - ESPCI - UPMC Univ. Paris 06 - PDD Univ. Paris 07 \\ 10, rue Vauquelin 75231 Paris Cedex 5, France
}

(Received ?? and in revised form ??)

The stability of two-layer oscillatory flows was studied experimentally in a cylindrical container with a vertical axis. Two superposed immiscible liquids, differing largely in viscosity, were set in relative oscillatory motion by alternating container rotation. Waves arising beyond a threshold were observed in detail for small oscillation frequencies ranging from $0.1 \mathrm{~Hz}$ to $6 \mathrm{~Hz}$. Measurements were performed on the growth rate and the wavenumber of these waves. The instability threshold was determined from the growth rate data. It was found that the threshold and the wavenumber varied with the frequency. In particular, significantly lower thresholds and longer waves were found than those predicted by the inviscid theory of the oscillatory Kelvin-Helmholtz instability. Favourable agreement with the predictions of an existing viscous theory for small oscillation amplitude flows indicates the important role of viscosity, even at the highest frequency, and suggests a similar mechanism behind the instability as the short wave instability in steady Couette flows. A semi-numerical stability determination for finite amplitude flows was also performed to improve the prediction in experiments with a frequency lower than $1 \mathrm{~Hz}$.

Key words: Boundary layer stability; Pattern formation; Coastal engineering

\section{Introduction}

The stability of two-layer flows is of practical importance and has been studied by many researchers in a variety of configurations (Joseph \& Renardy 1992). However, the configuration of oscillatory shearing flows has been less well studied than other configurations. Inviscid theories developed by Kelly (1965) and Lyubimov \& Cherepanov (1987) predict an instability that has the same threshold in relative velocity, $\Delta U_{c r}$, and the same most unstable wavenumber mode, $k_{c r}$, as the classical Kelvin-Helmholtz instability. The following predictions are given for oscillatory flow:

$$
\Delta U_{c r}=\sqrt{\frac{2\left(\rho_{1}^{2}-\rho_{2}^{2}\right) g \lambda_{c a p}}{\pi \rho_{1} \rho_{2}}}, \quad k_{c r}=\frac{2 \pi}{\lambda_{c a p}}
$$

$\dagger$ Present address: Laboratoire J.-A. Dieudonné, Université de Nice Sophia-Antipolis, Parc Valrose - 06108 Nice Cedex 2, France. 


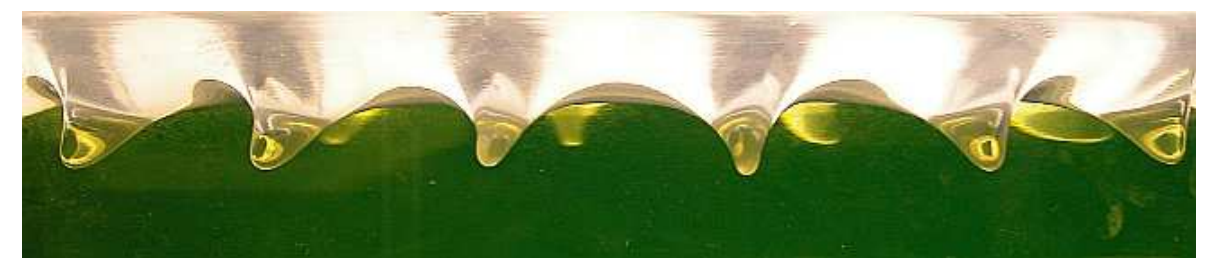

FIgURE 1. Waves formed at a silicone oil-water interface along a cylindrical container periphery (side view, the frequency and amplitude of container rotation are $\omega / 2 \pi=4.0 \mathrm{~Hz}$ and $\Phi_{0}=2.5$ degrees).

where $\rho_{1}\left(\rho_{2}\right)$ is the density of the lower (upper) fluid, and $\lambda_{\text {cap }}$ is the capillary wavelength $\lambda_{\text {cap }}=2 \pi \sqrt{\gamma /\left(\rho_{1}-\rho_{2}\right) g}$ ( $\gamma$ : interfacial tension, $g$ : gravitational acceleration).

The formation of interface waves, called "frozen waves", was observed experimentally in oscillatory two-layer flows (Wolf 1969; Shyh \& Munson 1986; Beysens et al. 1998; Ivanova et al. 2001a,b; Talib et al. 2007). These experiments were typically conducted in a container with two superposed immiscible liquid layers. The container was vibrated horizontally to induce a shearing motion between the liquid layers (for more details on the existing experiments, see Yoshikawa \& Wesfreid 2010). Experimental values of $\Delta U_{c r}$ and $k_{c r}$ showed significant dependence on the oscillation frequency (Beysens et al. 1998; Ivanova et al. 2001a,b). Furthermore, $\Delta U_{c r}$ is often much lower than the predicted values (1.1). Recent theoretical investigations have shown that the inclusion of viscosity in the linear stability analysis can explain this behaviour (Talib et al. 2007; Yoshikawa \& Wesfreid 2010).

In our previous paper (Yoshikawa \& Wesfreid 2010, hereafter, referred to as Y\&W), we developed a linear stability theory for viscous flows. An asymptotic case, in which the oscillation amplitude $\Delta U / \omega$ ( $\Delta U$ : relative velocity between two fluids) is much smaller than the wavelength $\lambda$, was considered. The stability was determined with using asymptotic expansions about a small Keulegan-Carpenter number $K=2 \Delta U / \omega \lambda$. This number is twice the ratio of the oscillation amplitude to the wavelength and reflects the importance of advection relative to local variation in time-periodic flows. This viscous smallamplitude theory showed an important dependence of $\Delta U_{c r}$ and $k_{c r}$ on the frequency, as found in the experiments. A particular frequency regime, called the moderate frequency regime, was distinguished for layers with a large viscosity contrast, where the instability threshold becomes substantially lower. In this regime, it was suggested that instability was due to perturbation flow generated through the velocity continuity at the disturbed interface, similar to the short wave instability of steady two-layer Couette flows (Hooper \& Boyd 1983; Hinch 1984). Comparison with the experiments of Talib et al. (2007) with large frequencies $(10 \mathrm{~Hz}<\omega / 2 \pi<50 \mathrm{~Hz})$ indicated that the asymptotic theory is valid up to a relatively large value of $K(\approx 0.5)$.

In the present paper, we report detailed observations of the evolution of interface waves developed at the interface of highly viscous silicone oil and water (see figure 1). The instability threshold and wavenumber of the resulting patterns are also reported. Experiments were carried out with a vertically installed cylindrical container in which the two fluids were superposed. In contrast with most of the preceding experiments, the relative oscillatory motion of the fluid layers is in the azimuthal direction. It was created by the shear force exerted by the container sidewall on the fluid layers. The frequencies are smaller than in typical experiments and range from $0.1 \mathrm{~Hz}$ to $6 \mathrm{~Hz}$. Only Shyh \& Munson (1986) performed experiments in this geometry. They reported wave formation 


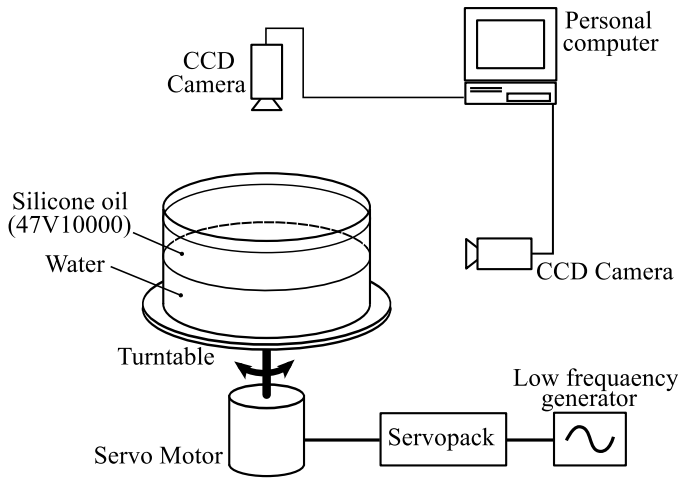

FIGURE 2. Schematic illustration of an experimental set-up.

through a supercritical bifurcation. Although they measured the instability threshold and the height of saturated waves, they did not report any wavelength measurement.

The set-up and the basic flows established in the absence of interface waves are described in section 2. Experimental results, covering a wide range of $K(0.2<K<1.5)$, are presented and discussed in section 3, including a comparison with the small-amplitude theory of Y\&W. In section 4, a semi-numerical determination of the linear stability is performed to provide better predictions for large $K$ experiments (equivalently, for small frequency experiments). Conclusions are given in section 5 .

\section{Experimental set-up}

\subsection{Set-up}

Highly viscous silicone oil (47V10000) of density $\rho_{2}=973 \mathrm{~kg} / \mathrm{m}^{3}$ and viscosity $\nu_{2}=$ $10000 \mathrm{~mm}^{2} / \mathrm{s}$ and water were superposed in a cylindrical glass container with a vertical axis as shown in figure 2. The container radius $R$ was $112 \mathrm{~mm}$ or $120 \mathrm{~mm}$. The layer thicknesses were $35 \mathrm{~mm}$ for the oil and about $70 \mathrm{~mm}$ for the water. Sodium dodecyl sulphate (SDS) was dissolved in the water at a concentration of $1.8 \mathrm{~kg} / \mathrm{m}^{3}$ to minimize the undesired optical effects of the interface meniscus during the interface visualization in lateral views. A small amount of fluorescein was also added to the water to enhance the optical contrast at the interface. The interfacial tension of this solution with the silicone oil was measured as $10 \pm 3 \mathrm{mN} / \mathrm{m}$ (correspondingly, $\lambda_{\text {cap }}=39 \pm 6 \mathrm{~mm}$ ) by the pendant drop method.

The container is mounted on a horizontal turntable that executes alternating rotations around a vertical axis aligned with the container centre axis. The angle $\Phi$ of the turntable rotation follows $\Phi=\Phi_{0} \sin (\omega t)$ for a given frequency $\omega / 2 \pi$ and a given rotation amplitude $\Phi_{0}$. When the container is in rotation, the sidewall shears the fluids and sets them in motion. In a fluid with kinematic viscosity $\nu$, such oscillatory shears penetrate over a depth on the order of the Stokes boundary layer thickness $\delta=\sqrt{2 \nu / \omega}$. In the oil, this penetration depth, $\delta_{2}$, is comparable to the container radius, and the entire layer is set in motion. In water, the depth $\delta_{1}$ is quite small (less than $1.8 \mathrm{~mm}$ in our experiments) and the water layer is primarily at rest in the laboratory frame. Therefore, the essential configuration is that in which a viscous oil layer shears a stationary water layer in an oscillatory manner, generating the condition for the oscillatory Kelvin-Helmholtz instability.

A circular fluorescent lamp is used for lighting the oil-water interface uniformly around the container periphery. The interface behaviour is observed optically from the top and 


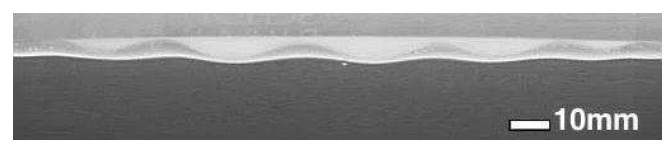

Linear evolution

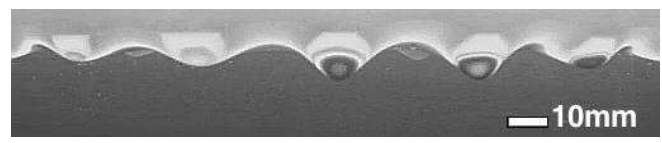

Nonlinear amplification

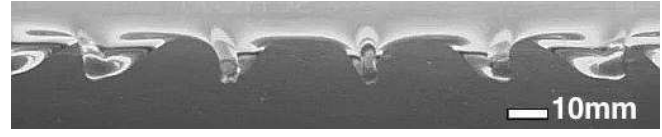

Final saturated state

(a)

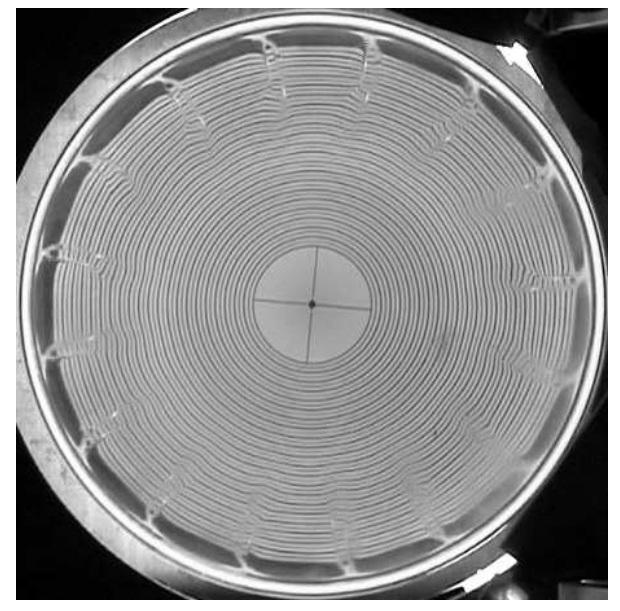

(b)

FiguRE 3. Wave evolution from a lateral view (a) and a final saturated state from a top view (b). A movie of the evolution shown in (a) can be found on the Internet at the Journal of Fluid Mechanics website (the oscillation parameters: $\omega / 2 \pi=1.10 \mathrm{~Hz}$ and $\Phi_{0}=6.7$ degrees).

side views by two CCD cameras (see pictures (a) and (b) in figure 3 for side and top view images). The optical axis of the top-view camera aligns with the container's centre axis, while that of the side-view camera is set to see the interface in a horizontal radial direction at the interface level. The side- and top-view cameras observe, respectively, the vertical and radial evolutions of interface deformations. To enhance the visualization of interface deformations in the top view, concentric circles are printed on the container bottom, as seen in image (b). Images captured by both cameras are recorded by the computers for analysis.

\subsection{Basic flow}

Flows induced by alternating container rotation can be calculated by assuming a flat oil-water interface with an infinite viscosity contrast. A one-dimensional flow $\boldsymbol{U}=U \boldsymbol{e}_{\varphi}$ $\left(\boldsymbol{e}_{\varphi}\right.$ : azimuthal unit vector) in a fluid of a viscosity $\nu$ is governed by the Navier-Stokes equation in cylindrical coordinates $(r, \varphi, z)$ :

$$
\frac{\partial U}{\partial t}=\nu\left[\frac{1}{r} \frac{\partial U}{\partial r}+\frac{\partial^{2} U}{\partial r^{2}}+\frac{\partial^{2} U}{\partial z^{2}}-\frac{U}{r^{2}}\right]
$$

For the oil layer $(z>0)$, the shear at the interfaces with air and water is negligible. The flow in the oil is induced only by the shear exerted by the sidewall and is uniform in the $z$ direction. The periodic flow satisfying (2.1) and the no-slip condition on the wall is

$$
\begin{gathered}
U_{2}=\left|\hat{U}_{2}\right| \cos \left(\omega t+\arg \hat{U}_{2}\right) \\
\text { with } \hat{U}_{2}(r)=\omega R \Phi_{0} \frac{J_{1}\left(\sqrt{-i 2} r / \delta_{2}\right)}{J_{1}\left(\sqrt{-i 2} R / \delta_{2}\right)}
\end{gathered}
$$

where $J_{1}(x)$ is the Bessel function of first kind of order 1 . The magnitude $\left|\hat{U}_{2}\right|$ is an increasing function of $r$ and attains its maximum value, $\omega R \Phi_{0}$, at the wall.

In the water $(z<0)$, the flow is induced by the shear exerted by the oil layer as well as by the sidewall. The velocity field is no longer uniform in the $z$-direction. A Stokes 
boundary layer will develop on the oil-water interface. The solution of equation (2.1) that satisfies the no-slip conditions on the wall and at the interface can be expressed as a series of Bessel functions $\left\{J_{1}\left(\mu_{n} r / R\right)\right\}(n=1,2, \cdots)$ with $z$-dependent coefficients $\left(\mu_{n}\right.$ : $n^{\text {th }}$ zero of $\left.J_{1}(x)\right)$. Inspection of this solution for a thick water layer, as in the experiment, shows that the velocity field is well approximated by the following equation at a radial position smaller than $R-5 \delta_{1}$ :

$$
U_{1}=\left|\hat{U}_{2}\right| e^{z / \delta_{1}} \cos \left(\omega t+\frac{z}{\delta_{1}}+\arg \hat{U}_{2}\right)
$$

In the vertical direction, this flow has the same velocity profile as in Stokes' oscillatory flow induced by an oscillating flat plate (Lamb 1945, art. 345). It diminishes rapidly with the distance from the interface. The water layer is immobile. On the sidewall, another Stokes boundary layer develops. With $r$ increasing in a thin region $R-5 \delta_{1}<r<R$, the velocity $U_{1}$ approaches the sidewall velocity. Consequently, the relative velocity between the oil and water layers goes to zero.

As shown in section 4, the determined flow, (2.2) and (2.4), at a given radial position has the same velocity profile as in the flow considered in $\mathrm{Y} \& \mathrm{~W}$ in the limit of a large viscosity contrast. The stability of flows induced by container rotation can therefore be analyzed by using the formulation in $\mathrm{Y} \& \mathrm{~W}$. In the application of stability theories of two-dimensional flows in plane geometry (e.g., the inviscid theory (1.1) and the theory of $\mathrm{Y} \& \mathrm{~W})$ to the present experiment, the flow at $r \approx R-5 \delta_{1}$ should be considered, where the relative motion is maximum and the instability first occurs. The relative velocity between the layers at this radial position is well-approximated by $\Delta U=R \omega \Phi_{0}$, because the Stokes boundary layer in water is very thin $\left(\delta_{1} \ll R\right)$ in the present experiment. The validity of the theoretical flow (2.2) and (2.4) was examined experimentally as described below. Centrifugal effects neglected in the derivation of (2.2) and (2.4) will be discussed later.

The theoretical basic flow (2.2) and (2.4) were confirmed by two tests: (i) following the positions of coloured water drops suspended in the oil layer and comparing them with the calculation and (ii) injecting dye into the water layer to qualitatively observe the immobility of the water layer. In test (i), the drops had a diameter of about $5 \mathrm{~mm}$, which was much smaller than the Stokes boundary layer thickness $\delta_{2}$ in the oil within the examined frequency range $(180 \mathrm{~mm}$ for $0.1 \mathrm{~Hz}$ and $23 \mathrm{~mm}$ for $6 \mathrm{~Hz})$. Viscous drag exerted on a drop is then dominant over the inertia of the drop. Therefore, the motions of the drops directly represent the flow in the oil layer. It was observed that the drops moved sinusoidally in the azimuthal direction like $\varphi=\phi_{\mathrm{m}} \sin (\omega t+\theta)$, without any radial displacement. Figure 4 shows the amplitude $\phi_{\mathrm{m}}(r)$ and the phase $\theta(r)$ of the flow determined from the drop motions and compares them with theoretical predictions of

$$
\int U_{2} d t=\frac{\left|\hat{U}_{2}\right|}{\omega} \sin \left(\omega t+\arg \hat{U}_{2}\right)
$$

The theoretical predictions are found to agree well with the experiments. In test (ii), a small amount of dye (fluorescein solution) was injected at a middle level of the water layer over a wide horizontal area. During a number of container rotations at different frequencies, the traces of dye were observed to be immobile except near the container wall and the interface with the oil, implying the immobility of the bulk of the water layer.

Centrifugal effects were not taken into account in the derivation of the flow (2.2) and (2.4). In experiments, a dome-like global deformation of the oil-water interface was observed, as also reported by Shyh \& Munson (1986). This deformation is caused by a 


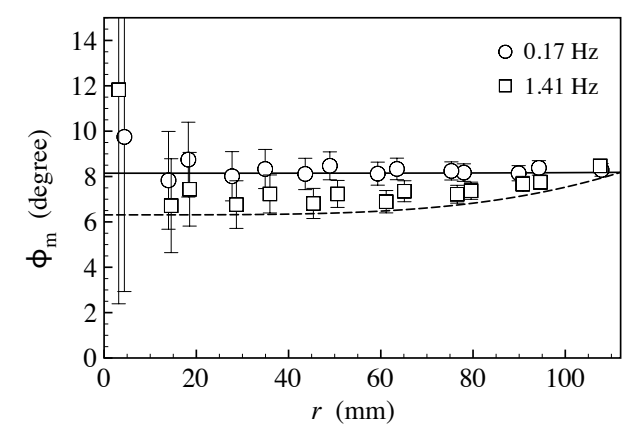

(a)

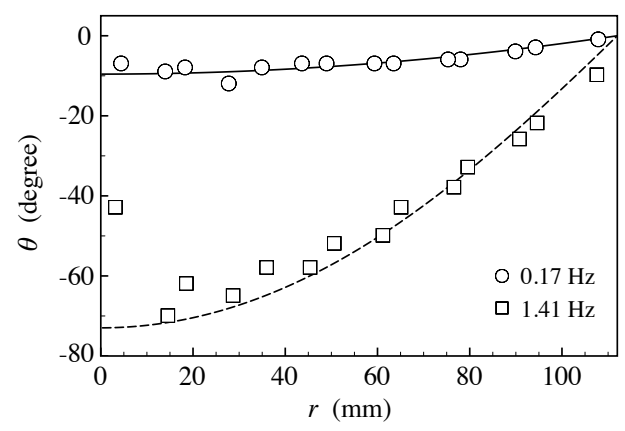

(b)

Figure 4. Azimuthal motion of drops suspended in the oil layer: $\varphi=\phi_{\mathrm{m}} \sin (\omega t+\theta)$. The circles and the squares stand for $0.17 \mathrm{~Hz}$ and $1.41 \mathrm{~Hz}$, respectively. $\left(R=112 \mathrm{~mm}\right.$ and $\Phi_{0}=8.2$ degrees for both experiments.) The lines show the prediction of equation (2.5).

pressure increase $\Delta p_{c e n}=\int \rho_{2}\left(U_{2}^{2} / r\right) d r$ in the oil due to centrifugal force. The interface should shift vertically in order to balance $\Delta p_{c e n}$ at the interface by a hydrostatic pressure. The dome height $H_{D}$ can be estimated by this balance: since $\Delta p_{c e n} \sim \rho_{2}\left(R \omega \Phi_{0}\right)^{2} / 4$, the height $H_{D}$ is given approximatively by $\rho_{2}\left(R \omega \Phi_{0}\right)^{2} / 4\left(\rho_{1}-\rho_{2}\right) g$. The latter value should be small compared with the container diameter $2 R$ for a flat oil-water interface. Centrifugal force also generates a radial flow component $u_{r}$ in the boundary layer on the oil-water interface in water. This component can be estimated by the theoretical results of Rogers \& Lance (Greenspan 1969): $u_{r}=-0.886\left(\nu_{1} \partial \Phi / \partial t\right)^{1 / 2}$. For one-dimensional velocity fields in the azimuthal direction, the component $u_{r}$ should be small compared with the azimuthal velocity $R \omega \Phi_{0}$. From these two requirements, $H_{D} \ll 2 R$ and $u_{r} \ll R \omega \Phi_{0}$, one can derive the following conditions on the container velocity $R \omega \Phi_{0}$ for negligible centrifugal effects:

$$
\frac{0.79 \nu_{1}}{R} \ll R \omega \Phi_{0} \ll \sqrt{\frac{8\left(\rho_{1}-\rho_{2}\right) g R}{\rho_{1}}}
$$

It follows that a large container radius is necessary for proper experiments. For example, the critical velocity is typically on the order of $\Delta U_{c r}$ given by equation (1.1). Using $R \omega \Phi_{0} \sim \Delta U_{c r}$ in the conditions (2.6), one will find $R \gg \sqrt{\rho_{1} \rho_{2} \nu_{1}^{2} /\left(\rho_{1}^{2}-\rho_{2}^{2}\right) g \lambda_{\text {cap }}}$ and $R \gg\left(\rho_{1}+\rho_{2}\right) \lambda_{\text {cap }} / 4 \pi \rho_{2}$. In the present experiments, the conditions (2.6) are well satisfied. Indeed, $H_{D} / 2 R<5 \times 10^{-3}$ and $u_{r} / R \omega \Phi_{0} \lesssim 0.05$ for all the measurements presented in the next section.

\section{Results \& discussion}

\subsection{Wave evolution}

When oscillatory rotations of the container exceed a certain threshold, the interface deforms waves along the container periphery, where the relative oscillatory motion of the layers is the most intense. These waves are fixed on the oil layer and sheared by water and have a radial extension of a few centimeters, fading out over this extension towards the container centre. No waves form inside a circular region at the centre of the interface. At the beginning of their emergence, the shape of the waves along the periphery is sinusoidal (see the first picture in figure 3 (a)). The wave height $h$, defined as the difference between the crest and trough levels in the lateral view, increases exponentially with time at this linear evolution stage. In figure 5 (a), the temporal evolutions of $h$ are shown in a logarithmic scale for different oscillation parameters. The initial linear parts of these curves correspond to the linear evolution stage. After attaining around 3-4 mm, 


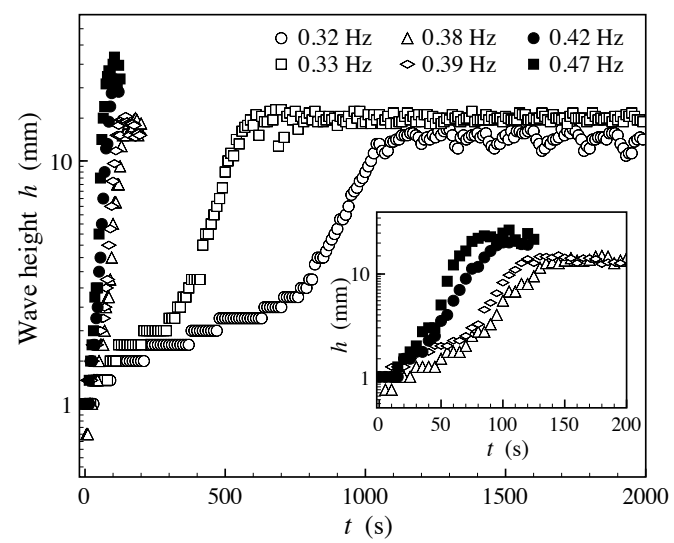

(a)

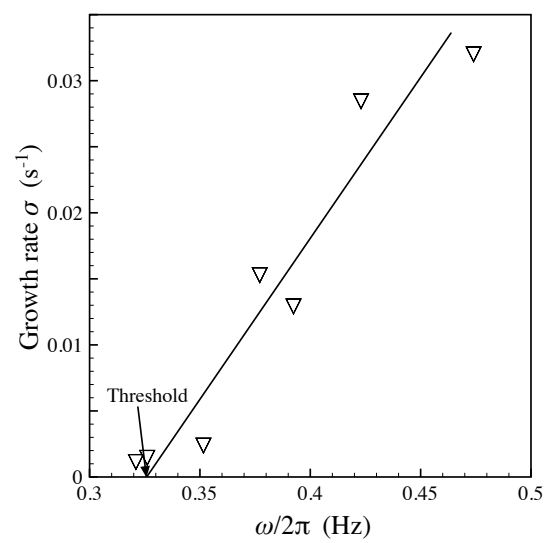

(b)

FiguRE 5. Wave height evolutions (a) and growth rates (b) for different frequencies. The oscillation amplitude of the container is fixed at 25 degrees $(R=112 \mathrm{~mm})$. In (a), the evolutions for frequencies higher than $0.39 \mathrm{~Hz}$ are enlarged in the small inserted plot.

the height is found to increase more rapidly, as seen in the second linear section of the evolution curves in the figure. At this nonlinear amplification stage, the formation of non-sinusoidal waves start from some locations and propagate along the periphery. The waves exhibit finger-like invading parts of the oil into the water (see the second picture in figure $3(\mathrm{a})$ ). The radial extension of the waves continues to increase. This nonlinear amplification continues until the wave height arrives at a maximum value. Then, the waves begin to relax to their final saturated state. When the frequency is relatively small $(\omega / 2 \pi \lesssim 3 \mathrm{~Hz})$, thinning of the oil fingers is observed during the relaxation. Finally, being sheared by the stationary water, the oil fingers horizontally oscillate like pendulums with their roots fixed on the oil layer. This thinning process is often accompanied by a small reduction of the height. A link to the movie for the entire evolution process in this smallfrequency experiment is found on the Journal of Fluid Mechanics website. In experiments with a large frequency $(\omega / 2 \pi \gtrsim 3 \mathrm{~Hz})$, the thinning process is not observed and the final wave shape is different from that of small-frequency experiments, as seen in figure 1. The waves are more triangular in shape, as reported by Ivanova et al. (2001a), and look "frozen" after saturation without pendulum-like oscillations. Only the tips of the oil fingers oscillate with a small amplitude.

Jalikop \& Juel (2009) investigated saturated waves generated in oscillatory two-layer flows, by experiments in a horizontally vibrated parallelepiped container. The considered upper-layer fluids were silicone oils of viscosities $114 \mathrm{~mm}^{2} / \mathrm{s}$ and $210 \mathrm{~mm}^{2} / \mathrm{s}$. The oscillation frequencies ranged from 20 to $30 \mathrm{~Hz}$. They distinguished gravity-dominated waves and capillary-dominated waves. The former arise in the weakly nonlinear regime and characterised by small curvature $\left(\lesssim 2 \pi / \lambda_{\text {cap }}\right)$ at the crest or trough and by small wave height $\left(\lesssim \lambda_{\text {cap }} / 2 \pi\right)$. They exhibit trochoid or inverted-trochoid shapes, depending on the viscosity $\nu_{2}$ of the upper-layer fluid. The latter waves are observed in the strongly nonlinear regime and have sharp crests and troughs. In the present experiments, the curvature at the crest is much smaller than $2 \pi / \lambda_{\text {cap }}\left(=0.16 \mathrm{~mm}^{-1}\right)$. For the waves formed at $4 \mathrm{~Hz}$ shown in figure 1 , for example, the curvature at the crest is around $0.06 \mathrm{~mm}^{-1}$. Indeed, all the experiments were performed with oscillation parameters near the instability thresholds. The observed waves are gravity-dominated. However, the waves attain larger height than $\lambda_{c a p} / 2 \pi(=6.2 \mathrm{~mm})$, as seen in figure 5 (a). Jalikop \& Juel (2009) reported the wave height increases with frequency decreasing. Their data also indicate a wave height 
increase with the viscosity $\nu_{2}$. Both tendencies would lead to the large gravity-dominated waves observed in the present experiments. Another remarkable difference of the present waves from Jalikop \& Juel (2009) is their shape. At large frequencies (>3 Hz), the oil fingers have sharp tips and waves exhibit non-trochoidal shapes (e.g., figure 1). Waves with the deep thin fingers observed in small-frequency experiments $(<3 \mathrm{~Hz})$ are also non-trochoidal (e.g., the third picture in figure 3 (a)). The shape difference would also stem from the large viscosity and small frequencies. The sharp tips and the deep thin fingers are formed during their horizontal oscillation in the stationary water. The size $L$ of oscillating tips ought to be smaller than $\delta_{2}$, as the viscous relaxation time $\left(\sim \sqrt{L^{2} / \nu_{2}}\right)$ should be smaller than the oscillation time scale $\omega^{-1}$ for their oscillatory motion. In the preceding experiments (Ivanova et al. 2001a; Jalikop \& Juel 2009), $\delta_{2}$ is on the order of a few millimetres. The size $L$ is then on the order of one millimetre. However, oscillation of such small structures will be prevented by interface capillarity. The sharp tips will not be formed. In the present experiments, $\delta_{2}$ ranges from $23 \mathrm{~mm}$ to $180 \mathrm{~mm}$ due to a large $\nu_{2}$ and small frequencies. Alternative shear exerted by water can therefore set the tips in oscillation and sharpen them. Particularly, $\delta_{2}$ becomes comparable with the wavelength below $3 \mathrm{~Hz}$. Entire fingers can oscillate at such small frequencies and the deep thin fingers are formed.

A wave height evolution similar to figure 5 (a) is found in the formation of sand ripples under oscillatory water flow (Stegner \& Wesfreid 1999). One similarity between the two systems is that a linear evolution is followed by a rapid nonlinear amplification in both scenerios. In the ripple formation, the latter stage corresponds to a transition to ripples with sharp crests, called vortex ripples. Vortex ripples are associated with flow separation behind the crests. The criterion of Sleath (1984), often used to predict the presence of the separation, is that the ratio of the ripple height to the wavelength is larger than around 0.1 . In the two-fluid experiment, the nonlinear amplification also begins when this criterion is satisfied. Indeed, the above-mentioned starting height is around 0.1 times larger than the capillary wavelength, which is a typical wavelength as seen below. The nonlinear evolution of waves and an analogy to the sand ripple formation are interesting subjects to investigate but are beyond the scope of the present work. A possible influence of the contact line dynamics on the nonlinear evolution of waves is as well. They will be the subjects of future publications.

\subsection{Stability $\&$ wavenumber selection}

The linear growth rate $\sigma$ of interface waves is determined as the slope of semilog-plotted height evolutions at the linear evolution stage. A series of measurements of the growth rate with a fixed oscillation amplitude for different frequencies gives an estimate of the instability threshold in frequency by linear extrapolation, as shown in figure 5 (b). Determined thresholds for different amplitudes yield a stability diagram on the amplitudefrequency plane, as shown in figure 6 (a). The rotation amplitude $\Phi_{0}$ has been transformed into the amplitude of azimuthal oscillation of the container at the sidewall, $R \Phi_{0}$, where the first wave formation is observed. It is seen that the threshold lowers monotonically as the frequency increases. The stability diagram is shown in the velocity-frequency plane in the inset of the figure (The velocity $\Delta U$ is calculated by $\Delta U=\omega R \Phi_{0}$ ). It is seen that the instability threshold is characterized by a constant velocity over a wide frequency range. Its value, around $92 \mathrm{~mm} / \mathrm{s}$, is substantially smaller than the prediction of the inviscid theory (1.1), which gives $115 \mathrm{~mm} / \mathrm{s}$. For frequencies lower than $1 \mathrm{~Hz}$, the critical velocity $\Delta U_{c r}$ diverges. Similar behaviour of the instability threshold was reported in the 


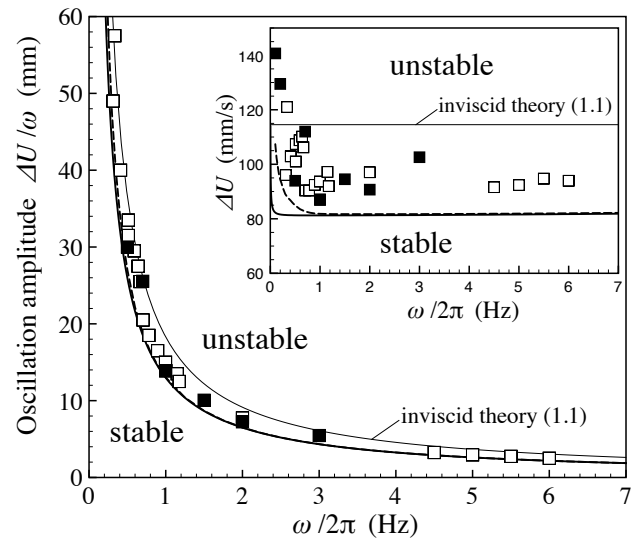

(a) Stability diagram

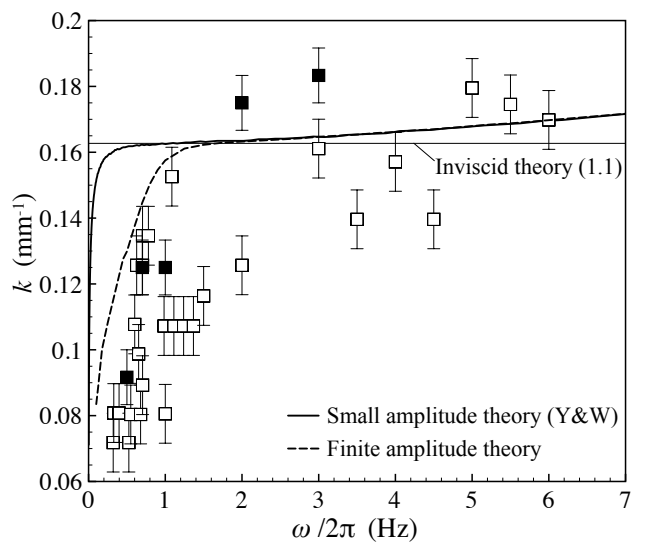

(b) Wavenumber of the most unstable mode

Figure 6 . Stability diagram and the most unstable mode. Experimentally obtained values ( $\square$ : with a container of $R=112 \mathrm{~mm}$, $\mathbf{\square}$ : with a container of $R=120 \mathrm{~mm}$ ) are compared with the viscous small-amplitude theory of $\mathrm{Y} \& \mathrm{~W}$ (bold solid line) and the viscous finite-amplitude theory presented in section 4 (dash line).

literature (Ivanova et al. 2001a,b; Talib et al. 2007), where the authors employed fluids of high viscosity.

The oil viscosity affects the interface stability, as expected from its large value. According to the analysis in $\mathrm{Y} \& \mathrm{~W}$, very high frequencies are required for neglecting viscous effects: $\omega / 2 \pi>10^{4} \cdot \nu_{2} / \pi \lambda_{\text {cap }}^{2} \approx 20 \mathrm{kHz}$. In the figure, the prediction of the viscous smallamplitude theory of $\mathrm{Y} \& \mathrm{~W}$ is shown as a bold solid line. This theory predicts a constant critical velocity for frequencies higher than $0.12 \mathrm{~Hz}$. An experiment with such a frequency falls in the moderate frequency regime distinguished in $\mathrm{Y} \& \mathrm{~W}$. In this regime, the critical velocity is smaller than that of the inviscid theory $(1.1)$ by a factor $\sqrt{\rho_{1} /\left(\rho_{1}+\rho_{2}\right)}=0.507$. The viscous theory reproduces the observed constant critical velocity in experiments with a frequency higher than $1 \mathrm{~Hz}$ and yields a diverging behaviour of $\Delta U_{c r}$ for small-frequency experiments $(<1 \mathrm{~Hz})$. However, it underestimates the threshold in these small-frequency experiments and does not provide an adequately rapid divergence of $\Delta U_{c r}$ with decreasing frequency, compared to the experimental values.

In experiments, the number $N$ of formed waves did not change with time. It was observed that the waves emerging from the periphery $(r=R)$ kept their relative azimuthal positions throughout their entire evolution. The primary wavenumber $k(=2 \pi / \lambda)$ can then be calculated from the number $N$ of formed waves in the saturated state by the relation $k=N / R$. In figure $6(\mathrm{~b})$, this wavenumber is shown as a function of the frequency. Error bars in the figure represent the effect of geometrical confinement, or quantification. The quantification gives \pm 1 uncertainty in the number of waves. It is seen that the wavenumber is smaller than the capillary wavenumber $2 \pi / \lambda_{\text {cap }}$ below around $2 \mathrm{~Hz}$. Observations of waves of $k$ smaller than the capillary wavenumber were also reported in the literature (Ivanova et al. 2001a,b; Talib et al. 2007). The wavenumber increases with frequency and seems to equal the capillary wavenumber beyond $2 \mathrm{~Hz}$.

The predictions of the viscous small-amplitude theory are plotted as a bold solid line in the figure. It shows an increasing behaviour of $k$ as increasing frequency. Above $0.2 \mathrm{~Hz}$, the predicted wavenumber is nearly constant and equal to the capillary wavenumber. The continuous slight increase beyond this value with increasing frequency is due to a peculiar behaviour found in $\mathrm{Y} \& \mathrm{~W}: k$ attains a maximum when $\left(\lambda_{\text {cap }} / \delta_{2}\right)^{2} \approx 60$ (equivalently 


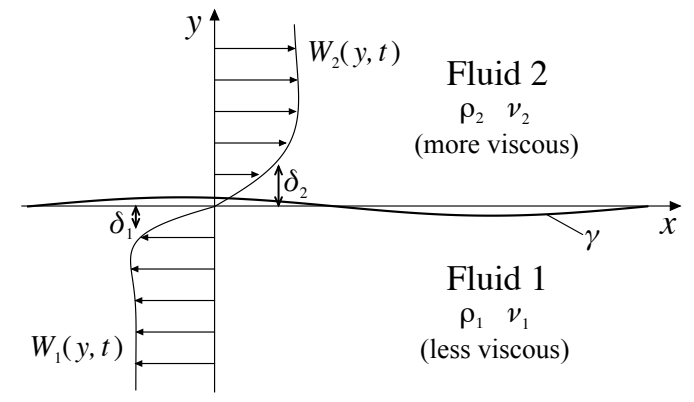

Figure 7. Oscillatory flow in semi-infinite fluid layers considered in Y\&W (in the interface reference frame). The basic flow, $W_{1}$ and $W_{2}$, are given in equation (4.1).

$\omega / 2 \pi \approx 126 \mathrm{~Hz}$, for the present silicone oil). Below $0.2 \mathrm{~Hz}$, the theory predicts a rapid decrease of $k$ with decreasing frequency. As in the comparison of the instability threshold, the viscous theory qualitatively explains the behaviour of the critical wavenumber. For large-frequency experiments $(>2 \mathrm{~Hz}$ ), it provides good quantitative predictions. However, the theory fails to reproduce quantitatively the experimental rapid decrease of $k$ with decreasing frequency below $2 \mathrm{~Hz}$.

In the above discussion, it was seen that the experimental results on the instability threshold and the critical wavenumber are explained by the viscous small-amplitude theory of $\mathrm{Y} \& \mathrm{~W}$ developed for flows in a plane geometry. As suggested in $\mathrm{Y} \& \mathrm{~W}$, the instability of oscillatory flows with frequency such that $\left(\lambda_{c a p} / \delta_{2}\right)^{2}<10 \rho_{2} / \rho_{1}$ (equivalently, $\omega / 2 \pi<20 \mathrm{~Hz}$, for the present silicone oil) is velocity-driven, i.e., due to perturbation flow generated through the velocity continuity at the disturbed interface, similar to the short wave instability of steady two-layer Couette flows (Hooper \& Boyd 1983; Hinch 1984). The generated perturbation flow is advected by the basic flow to yield out-of-phase flow component enhancing the interface disturbance. This mechanism is different from the classical Kelvin-Helmholtz instability (1.1), which is expected in very high-frequency experiments. Agreements with the viscous theory suggest that the present experiments concern the former velocity-driven instability, not the classical instability.

The viscous theory of $\mathrm{Y} \& \mathrm{~W}$ fails to quantitatively explain the small-frequency behaviour of $\Delta U_{c r}$ and $k_{c r}$. This is expected because the validity condition $K \lesssim 0.5$ of the small-amplitude theory is violated for these experiments. The effects due to a finite amplitude should be taken into account. In the next section, we solve the linear stability problem formulated in Y\&W without assuming small values for $K$. This solution enables one to distinguish amplitude effects from viscous ones on the stability and to improve quantitative predictions.

\section{Stability of flows with a finite oscillation amplitude}

We present a semi-numerical resolution method of the linear stability problem formulated in Section II of Y\&W. This formulation is for fluid layers of semi-infinite depth. Considered flows are two-dimensional and in plane geometry as seen in figure 7 . Note that the vertical coordinate is denoted by $y$ to prevent confusion with the cylindrical coordinate system used earlier. The flows have the following velocity field:

$$
\begin{cases}W_{1}=-\frac{\rho \kappa \Delta U}{1+\rho \kappa}\left[\cos (\omega t)-e^{\frac{y}{\delta_{1}}} \cos \left(\omega t+\frac{y}{\delta_{1}}\right)\right] & \text { (in the less viscous fluid) } \\ W_{2}=\frac{\Delta U}{1+\rho \kappa}\left[\cos (\omega t)-e^{-\frac{y}{\delta_{2}}} \cos \left(\omega t-\frac{y}{\delta_{2}}\right)\right] \quad \text { (in the more viscous fluid) }\end{cases}
$$


in the interface reference frame, i.e., the reference frame moving with the basic flow velocity $U_{\mathrm{i}}(t)$ at the interface level $(y=0)$. The density and viscosity contrasts, $\rho$ and $\kappa$, in equation (4.1) are $\rho=\rho_{2} / \rho_{1}$ and $\kappa=\sqrt{\nu_{2} / \nu_{1}}$. For fluids with a large viscosity contrast $(\kappa \gg 1)$, the above velocity profiles are reduced to $W_{1}=-\Delta U\left[\cos (\omega t)-e^{y / \delta_{1}} \cos (\omega t+\right.$ $\left.y / \delta_{1}\right)$ ] and $W_{2}=0$. These are identical to the velocity fields $(2.2)$ and (2.4) with $U_{\mathrm{i}}=$ $\left|\hat{U}_{2}\right| \cos \left(\omega t+\arg \hat{U}_{2}\right)$ and $\Delta U=\left|\hat{U}_{2}\right|$. The stability of the flow induced by container rotation can therefore be analyzed by using the formulation in $\mathrm{Y} \& \mathrm{~W}$.

The stability problem consists of linearized vorticity equations and boundary conditions with respect to stream functions and interface disturbance. These are decomposed into a net evolution $e^{\sigma t}$ and different frequency components according to the Floquet theory (Yih 1967). The boundary conditions required at the interface are the velocity continuity, stress balance and the kinematic equation of a material interface. In appendix A, these boundary conditions written in the interface reference frame are reproduced from $\mathrm{Y} \& W$. All the equations are nondimensionalized with the time scale $\omega^{-1}$ and the velocity scale $\Delta U$. Lengths in a fluid are scaled by the Stokes boundary layer thickness in the fluid to respect the dynamics inside the boundary layer. The resulting equations involve dimensionless parameters: wavenumber $q=\lambda_{\text {cap }} / \lambda$, frequency $\Omega=\left(\lambda_{\text {cap }} / \delta_{2}\right)^{2}$ and velocity parameter $\mathrm{B}=\pi \rho_{1} \rho_{2} \Delta U^{2} / 2\left(\rho_{1}^{2}-\rho_{2}^{2}\right) g \lambda_{\text {cap }}$, besides $\rho, \kappa$ and $K$.

\subsection{Resolution method}

The resolution method consists of a shooting resolution of the vorticity equation. The method was developed for calculating the oscillatory flow over a solid wavy wall (Vittori 1989) and was used to model the formation of sand ripples under sea waves (Vittori \& Blondeaux 1990). In application of this resolution, we consider only the situation corresponding to the experiments. We assume an interface disturbance having a static component and slow growth compared with the oscillation and viscous dissipation. It follows that the periodic components of flow can be calculated by neglecting their net evolution. Stability is then decided by a small steady component of the velocity at the interface.

The vorticity equation for the flow in water in the laboratory frame, where the water layer is immobile, is

$$
\left(\triangle_{1}-i 2 m\right) \triangle_{1} \widetilde{\phi}_{1, m}=i \pi K e^{y_{1}}\left[e^{i y_{1}}\left(\triangle_{1}-i 2\right) \widetilde{\phi}_{1, m-1}+e^{i y_{1}}\left(\triangle_{1}+i 2\right) \widetilde{\phi}_{1, m+1}\right]
$$

for $y_{1}<0(m=0, \pm 1, \pm 2, \cdots)$. $\widetilde{\phi}_{1, m}$ is the frequency component $e^{i m t}$ of the stream function in the laboratory frame. The vertical coordinate is nondimensionalized by $\delta_{1}$ and denoted by $y_{1}$. The basic flow concentrates only within the Stokes boundary layer developed on the interface $\left(y_{1}=0\right)$ and diminishes exponentially as $y_{1} \rightarrow-\infty$. The advection associated with the basic flow found in the right-hand side of equation (4.2), therefore diminishes with the distance from the interface. In the outer layer $y_{1}<-D$ ( $D$ represents the thickness of the boundary layer), the advection is negligible and the stream function is given by

$$
\widetilde{\phi}_{1, m}= \begin{cases}a_{1, m} e^{\frac{2 \pi q}{\kappa \sqrt{\Omega}} y_{1}}+b_{1, m} e^{\sqrt{\frac{4 \pi^{2} q^{2}}{\kappa^{2} \Omega}+i 2 m}} & (m \neq 0) \\ a_{1,0} e^{\frac{2 \pi q}{\kappa \sqrt{\Omega}} y_{1}}+b_{1,0} \frac{2 \pi q}{\kappa \sqrt{\Omega}} y_{1} e^{\frac{2 \pi q}{\kappa \sqrt{\Omega}} y_{1}} & (m=0)\end{cases}
$$

The constants $\left\{a_{1, m}, b_{1, m}\right\}(m=0, \pm 1, \pm 2, \cdots)$ will be determined by the boundary conditions, as seen later.

It is convenient to write the vorticity equation (4.2) for different frequency components 
in a single matrix equation:

$$
\frac{d \phi_{1}}{d y_{1}}=\boldsymbol{K}_{1}\left(y_{1}\right) \phi_{1}
$$

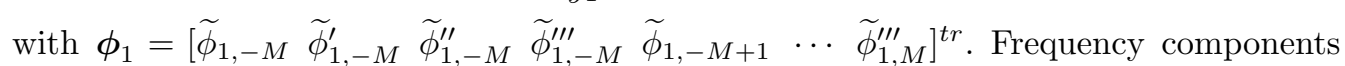
higher than $M$ have been truncated. For the definition of the matrix $\boldsymbol{K}_{1}$, see appendix B. After equation (4.3), the stream function $\phi_{1}$ in the outer layer can be written as:

$$
\phi_{1}=Q_{1}^{\infty} \boldsymbol{v}_{1}
$$

with a known matrix $\boldsymbol{Q}_{1}^{\infty}$ (for its explicit definition, see appendix B). The column vector

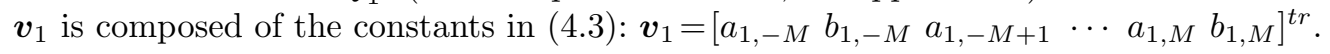

The flow inside the boundary layer is computed by numerically integrating the vorticity equation (4.4) from the boundary with the outer layer at $y_{1}=-D$. This integration yields a matrix function $\boldsymbol{Q}_{1}\left(y_{1}\right)$ which relates $\phi_{1}$ and $\boldsymbol{v}_{1}$ at an arbitrary $y_{1}$ by $\boldsymbol{\phi}_{1}=\boldsymbol{Q}_{1} \boldsymbol{v}_{1}$.

The computed stream functions $\left\{\widetilde{\phi}_{1, m}\right\}$ are used to derive the flow in the interface reference frame by a transformation: $\widetilde{\psi}_{1, m}=\sum_{n} J_{n-m}(\xi) \widetilde{\phi}_{1, n}$ (Rousseaux et al. 2004). $J_{n}(x)$ is the Bessel function of first kind of order $n$. The argument $\xi$ is associated with the amplitude of the relative oscillation between the reference frames $(\xi=-\pi K \rho \kappa /(1+\rho \kappa)$, for water layer). Introducing a matrix $\boldsymbol{J}_{1}$ representing this transformation, the stream function $\boldsymbol{\psi}_{1}$ in the new reference frame is found:

$$
\boldsymbol{\psi}_{1}=\boldsymbol{J}_{1} \boldsymbol{Q}_{1} \boldsymbol{v}_{1}
$$

where $\boldsymbol{\psi}_{1}$ is composed of $\left\{\widetilde{\psi}_{1, m}\right\}$ and their derivatives by a similar manner of the definition of $\phi_{1}$.

A similar procedure applied to the flow in the oil gives the stream function $\psi_{2}$ in the interface reference frame as

$$
\boldsymbol{\psi}_{2}=\boldsymbol{J}_{2} \boldsymbol{Q}_{2} \boldsymbol{v}_{2}
$$

where $\boldsymbol{\psi}_{2}, \boldsymbol{v}_{2}$ and $\boldsymbol{Q}_{2}$ are defined similarly to $\boldsymbol{\psi}_{1}, \boldsymbol{v}_{1}$ and $\boldsymbol{Q}_{1}$, respectively.

The boundary conditions at the interface can also be written in matrix notation:

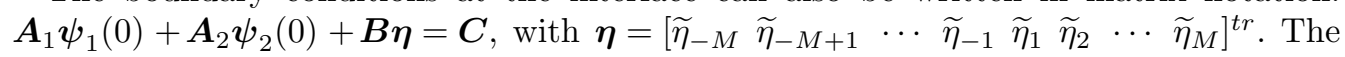
latter column vector does not include the static component of the interface disturbance $\widetilde{\eta}_{0}$. This component is set as $\widetilde{\eta}_{0}=1$ without any loss of generality. The column vector $\boldsymbol{C}$ is composed of terms associated with the interaction of $\widetilde{\eta}_{0}$ with the basic flow and terms due to the stabilization by gravity and capillarity. The definition of $\boldsymbol{C}$ and those of the matrices $\boldsymbol{A}_{1}, \boldsymbol{A}_{2}$ and $\boldsymbol{B}$ are found in appendix B. The above boundary condition in matrix form does not include the kinematic equation for the steady mode. The latter concerns the slow net evolution of the interface disturbance, and will be used to calculate the growth rate, as shown later (see equation (4.9)).

Substituting equations (4.6) and (4.7) into the boundary condition, one finds a linear algebraic equation set:

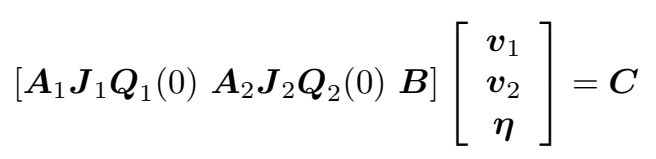

$\boldsymbol{v}_{1}$ and $\boldsymbol{v}_{2}$ computed by the inversion of the latter equation determine the flow $\boldsymbol{\psi}_{1}$ and $\boldsymbol{\psi}_{2}$ through (4.6) and (4.7).

In general, determined flow has a steady component of vertical velocity at the interface, 


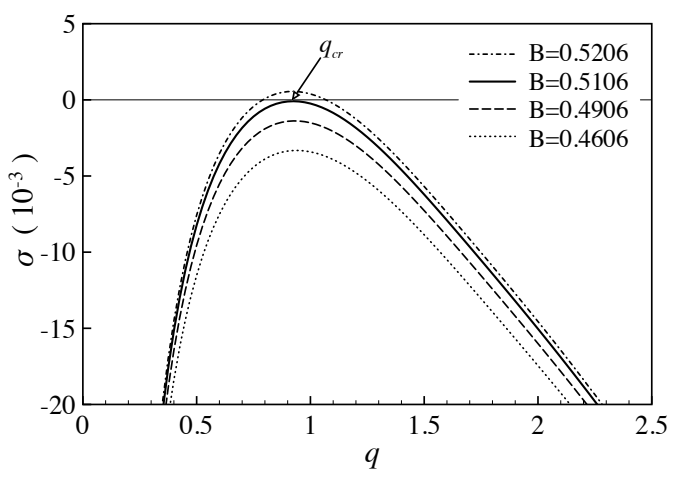

FigURE 8. Dispersion relation calculated by equation (4.9) for different values of B. Other parameters are $\rho=0.973, \kappa=100$ and $\Omega=0.3746$.

which yields a net growth or decay of interface disturbance. The growth rate is calculated by the kinematic equation for the steady mode:

$$
\sigma=-i \pi K \widetilde{\psi}_{1,0}(0)
$$

Figure 8 shows the dispersion relation computed by equation (4.9). In the calculation, the truncation of higher modes has been done typically at $M=20$. The boundary layer thickness has been set typically as $D=14$. It was confirmed that further increases of $M$ and $D$ did not change the calculation results. In the figure, it is seen that an increase of the control parameter B at a given frequency $\Omega$ leads to instability at a finite wavenumber $q$. At small and large wavenumbers, the system is stable due to the stabilizing effect of gravity and capillarity, respectively. The instability threshold and critical wavenumber is determined by the dispersion curve tangent to the $q$-axis.

\subsection{Results $\mathscr{E}$ discussion}

In figure 6 (a), the determined instability thresholds are shown by dash lines on both the amplitude-frequency and on the velocity-frequency planes. For frequencies larger than $1 \mathrm{~Hz}$, the result is coincident with the prediction of the small-amplitude theory, reproducing a constant critical velocity that is lower than the inviscid prediction (1.1). At small frequencies, where the small-amplitude theory failed to explain the experimental results quantitatively, a rapid divergence with decreasing frequency is predicted. It is quantitatively comparable with the experiments, implying that this diverging behaviour is due to finite $K$. As mentioned in section 3, the out-of-phase component of the perturbation vorticity generated by the velocity continuity at the interface would be responsible for the present instability. This mechanism could imply stabilization by overly-strong advection, as the advected vorticity is in-phase again.

In figure 6 (b), the predictions of the finite-amplitude analysis for the critical wavenumber are shown by dash lines. For frequencies higher than $1.6 \mathrm{~Hz}$, the predictions are coincident with the results of the small-amplitude theory and approximatively equal to the capillary wavenumber, as in the experiments. For lower frequencies, the finite-amplitude theory predicts a decrease in $k$ with a decrease in frequency, in quantitative agreement with the experiments, while the small-amplitude theory succeeded only in qualitative explanation of this behaviour. The difference between both viscous theories becomes significant for frequencies smaller than $1 \mathrm{~Hz}$.

The theory presented here provides good quantitative predictions on the threshold and the critical wavenumber over the entire frequency range examined experimentally. 
Failure of the preceding small-amplitude theory in quantitative predictions for smallfrequency experiments is due to the neglect of finite amplitude effects. Its inclusion is essential for flows with a frequency lower than $1 \mathrm{~Hz}$. It is interesting to calculate the Keulegan-Carpenter number for predicted waves at this frequency. After figure 6 (a), $\Delta U / \omega=13 \mathrm{~mm}$ and, according to figure $6(\mathrm{~b})$, the wavelength is equal to around $\lambda_{\text {cap }}$. It follows that $K \approx 2 \Delta U / \omega \lambda_{\text {cap }}=0.67$. This confirms the validity condition $K \lesssim 0.5$ of the small amplitude theory, which was proposed in $\mathrm{Y} \& \mathrm{~W}$.

\section{Conclusion}

Experiments were performed to study the stability of two-layer oscillatory flows with a large contrast in viscosity at the immiscible interface. The evolution of interface waves in the case of instability were observed in detail, and different evolution stages were distinguished. The instability threshold and the most unstable wavenumber mode were determined and compared with the viscous small-amplitude theory (Yoshikawa \& Wesfreid 2010). The theory reproduces the frequency-dependent behaviour of both threshold and wavenumber. For large-frequency experiments $(\omega / 2 \pi>1 \mathrm{~Hz})$, it also provided good quantitative predictions. This suggests that the observed waves are formed through a velocity-driven instability similar to the short wave instability of steady two-layer Couette flows (Hooper \& Boyd 1983; Hinch 1984). For small frequencies $(\omega / 2 \pi<1 \mathrm{~Hz}$ ), inclusion of the finite amplitude into the stability analysis is essential for correctly predicting the stability. The experiments agree well with the finite-amplitude theory over the entire frequency range.

The authors would like to thank Dr. A. Juel and Prof. T. Mullin for fruitful discussions and to acknowledge P. Jenffer, M. de Saint-Aubert and D. Renard for their support of our experiments.

\section{Appendix A. Boundary conditions at the interface for an arbitrary $K$}

These conditions are wrote in the interface reference frame. $\widetilde{\psi}_{1, m}$ and $\widetilde{\psi}_{2, m}$ are the frequency components $e^{i m t}$ of the stream functions in water and in oil, respectively. $\eta_{m}$ is the same frequency component of the interface disturbance. A prime on a function means differentiation with respect to the vertical coordinate.

Velocity continuity (tangent to the interface)

$$
\widetilde{\psi}_{1, m}^{\prime}-\widetilde{\psi}_{2, m}^{\prime}+\frac{\rho \kappa^{2}-1}{\rho \kappa^{2}}\left(\widetilde{W}_{1,1}^{\prime} \widetilde{\eta}_{m-1}+\widetilde{W}_{1,-1}^{\prime} \widetilde{\eta}_{m+1}\right)=0 \quad \text { at } y=0
$$

Velocity continuity (normal to the interface)

$$
\widetilde{\psi}_{1, m}-\kappa \widetilde{\psi}_{2, m}=0 \quad \text { at } y=0
$$

Stress balance (tangent to the interface)

$$
\begin{gathered}
\widetilde{\psi}_{1, m}^{\prime \prime}+\frac{4 \pi^{2} q^{2}}{\kappa^{2} \Omega} \widetilde{\psi}_{1, m}-\rho \kappa\left(\widetilde{\psi}_{2, m}^{\prime \prime}+\frac{4 \pi^{2} q^{2}}{\Omega} \widetilde{\psi}_{2, m}\right)+\frac{\kappa+1}{\kappa}\left(\widetilde{W}_{1,1}^{\prime \prime} \widetilde{\eta}_{m-1}+\widetilde{W}_{1,-1}^{\prime \prime} \widetilde{\eta}_{m+1}\right)=0 \\
\text { at } y=0
\end{gathered}
$$


Stress balance (normal to the interface)

$$
\begin{gathered}
\widetilde{\psi}_{1, m}^{\prime \prime \prime}-\left(\frac{12 \pi^{2} q^{2}}{\kappa^{2} \Omega}+2 \sigma+i 2 m\right) \widetilde{\psi}_{1, m}^{\prime}+i 2 \pi K\left(\widetilde{W}_{1,1}^{\prime} \widetilde{\psi}_{1, m-1}+\widetilde{W}_{1,-1}^{\prime} \widetilde{\psi}_{1, m+1}\right) \\
-\rho\left[\widetilde{\psi}_{2, m}^{\prime \prime \prime}-\left(\frac{12 \pi^{2} q^{2}}{\Omega}+2 \sigma+i 2 m\right) \widetilde{\psi}_{2, m}^{\prime}+i 2 \pi K\left(\widetilde{W}_{2,1}^{\prime} \widetilde{\psi}_{2, m-1}+\widetilde{W}_{2,-1}^{\prime} \widetilde{\psi}_{2, m+1}\right)\right] \\
-i \frac{\pi^{2} \rho K\left(1+q^{2}\right)}{\kappa(1+\rho) \mathrm{B} \sqrt{\Omega}} \widetilde{\eta}_{m}=0 \quad \text { at } y=0,
\end{gathered}
$$

Kinematic equation of a material interface

$$
i \pi K \widetilde{\psi}_{1, m}+(\sigma+i m) \widetilde{\eta}_{m}=0 \quad \text { at } y=0
$$

for $m=0, \pm 1, \pm 2, \cdots . \widetilde{W}_{1, \pm 1}$ and $\widetilde{W}_{2, \pm 1}$ are frequency components $e^{ \pm i t}$ of the basic flows $W_{1}$ and $W_{2}$, i.e., $W_{1}=\widetilde{W}_{1,1} e^{i t}+\widetilde{W}_{1,-1} e^{-i t}$ and $W_{2}=\widetilde{W}_{2,1} e^{i t}+\widetilde{W}_{2,-1} e^{-i t}$.

\section{Appendix B. Definitions of matrices}

For simple mathematical expressions, the following $\alpha_{1}, \alpha_{2}, Z_{1}$ and $Z_{2}$ are introduced.

$$
\alpha_{1}=\frac{2 \pi q}{\kappa \sqrt{\Omega}}, \quad \alpha_{2}=\frac{2 \pi q}{\sqrt{\Omega}}, \quad Z_{1}=\frac{\rho \kappa}{1+\rho \kappa} \quad \text { and } \quad Z_{2}=\frac{1}{1+\rho \kappa}
$$

A factor $\epsilon_{j}$ also used in the matrix definitions is $\epsilon_{j}=1$ for $j=1$ and $\epsilon_{j}=-1$ for $j=2$.

$\boldsymbol{K}$-matrix in the vorticity equation $(4.2)$ has a size of $(8 M+4)$-by- $(8 M+4)$. It is defined for flows in Fluid $j(j=1,2)$ as

$$
\boldsymbol{K}_{j}=\left[\begin{array}{ccccccc}
\boldsymbol{k}_{j,-M} & \boldsymbol{k}_{j,-M}^{+} & \boldsymbol{O} & \boldsymbol{O} & \cdots & \boldsymbol{O} & \boldsymbol{O} \\
\boldsymbol{k}_{j,-M+1}^{-} & \boldsymbol{k}_{j,-M+1} & \boldsymbol{k}_{j,-M+1}^{+} & \boldsymbol{O} & \cdots & \boldsymbol{O} & \boldsymbol{O} \\
\boldsymbol{O} & \boldsymbol{k}_{j,-M+2}^{-} & \boldsymbol{k}_{j,-M+2} & \boldsymbol{k}_{j,-M+2}^{+} & \cdots & \boldsymbol{O} & \boldsymbol{O} \\
\vdots & \vdots & \vdots & \vdots & \cdots & \vdots & \vdots \\
\boldsymbol{O} & \boldsymbol{O} & \boldsymbol{O} & \boldsymbol{O} & \cdots & \boldsymbol{k}_{j, M}^{-} & \boldsymbol{k}_{j, M}
\end{array}\right]
$$

with

$$
\begin{gathered}
\boldsymbol{k}_{j, m}=\left[\begin{array}{cccc}
0 & 1 & 0 & 0 \\
0 & 0 & 1 & 0 \\
0 & 0 & 0 & 1 \\
-\alpha_{j}^{2}\left(\alpha_{j}^{2}+i 2 m\right) & 0 & 2 \alpha_{j}^{2}+i 2 m & 0
\end{array}\right], \\
\boldsymbol{k}_{j, m}^{-}=\epsilon_{j} i \pi K Z_{j} e^{\epsilon_{j}(1+i) y_{j}}\left[\begin{array}{cccc}
0 & 0 & 0 & 0 \\
0 & 0 & 0 & 0 \\
0 & 0 & 0 & 0 \\
-\left(\alpha_{j}^{2}+i 2\right) & 0 & 1 & 0
\end{array}\right]
\end{gathered}
$$

and $\boldsymbol{k}_{j, m}^{+}=-\left(\boldsymbol{k}_{j, m}^{-}\right)^{*}$ for $m=0, \pm 1, \pm 2, \cdots$. The asterisk denotes the complex conjugate.

$\boldsymbol{Q}$-matrix associated with the flow in the outer layer is defined for Fluid $j(j=1,2)$ as:

$$
\boldsymbol{Q}_{j}^{\infty}=\left[\begin{array}{ccccc}
\boldsymbol{q}_{j,-M} & \boldsymbol{O} & \boldsymbol{O} & \cdots & \boldsymbol{O} \\
\boldsymbol{O} & \boldsymbol{q}_{j,-M+1} & \boldsymbol{O} & \cdots & O \\
\vdots & \vdots & \vdots & \cdots & O \\
O & O & O & \cdots & \boldsymbol{q}_{j, M}
\end{array}\right]
$$


with

$$
\begin{aligned}
\boldsymbol{q}_{j, 0}= & {\left[\begin{array}{cc}
e^{\epsilon_{j} \alpha_{j} y_{j}} & \alpha_{j} y_{j} e^{\epsilon_{j} \alpha_{j} y_{j}} \\
\epsilon_{j} \alpha_{j} e^{\epsilon_{j} \alpha_{j} y_{j}} & \alpha_{j}\left(1+\epsilon_{j} \alpha_{j} y_{j}\right) e^{\epsilon_{j} \alpha_{j} y_{j}} \\
\alpha_{j}^{2} e^{\epsilon_{j} \alpha_{j} y_{j}} & \alpha_{j}^{2}\left(2 \epsilon_{j}+\alpha_{j} y_{j}\right) e^{\epsilon_{j} \alpha_{j} y_{j}} \\
\epsilon_{j} \alpha_{j}^{3} e^{\epsilon_{j} \alpha_{j} y_{j}} & \alpha_{j}^{3}\left(3+\epsilon_{j} \alpha_{j} y_{j}\right) e^{\epsilon_{j} \alpha_{j} y_{j}}
\end{array}\right] \quad \text { and } } \\
\boldsymbol{q}_{j, m}= & {\left[\begin{array}{cc}
e^{\epsilon_{j} \alpha_{j} y_{j}} & e^{\epsilon_{j} \sqrt{\alpha_{j}^{2}+i 2 m} y_{j}} \\
\epsilon_{j} \alpha_{j} e^{\epsilon_{j} \alpha_{j} y_{j}} & \epsilon_{j} \sqrt{\alpha_{j}^{2}+i 2 m} e^{\epsilon_{j} \sqrt{\alpha_{j}^{2}+i 2 m} y_{j}} \\
\alpha_{j}^{2} e^{\epsilon_{j} \alpha_{j} y_{j}} & \left(\alpha_{j}^{2}+i 2 m\right) e^{\epsilon_{j} \sqrt{\alpha_{j}^{2}+i 2 m} y_{j}} \\
\epsilon_{j} \alpha_{j}^{3} e^{\epsilon_{j} \alpha_{j} y_{j}} & \epsilon_{j}\left(\alpha_{j}^{2}+i 2 m\right)^{3 / 2} e^{\epsilon_{j} \sqrt{\alpha_{j}^{2}+i 2 m} y_{j}}
\end{array}\right] \quad(m= \pm 1, \pm 2, \cdots) }
\end{aligned}
$$

The transformation matrix $\boldsymbol{J}_{j}(j=1,2)$ is

$$
\boldsymbol{J}_{j}=\left[\begin{array}{cccc}
J_{0}(\xi) \boldsymbol{E} & J_{1}(\xi) \boldsymbol{E} & \cdots & J_{2 M}(\xi) \boldsymbol{E} \\
J_{-1}(\xi) \boldsymbol{E} & J_{0}(\xi) \boldsymbol{E} & \cdots & J_{2 M-1}(\xi) \boldsymbol{E} \\
J_{-2}(\xi) \boldsymbol{E} & J_{-1}(\xi) \boldsymbol{E} & \cdots & J_{2 M-2}(\xi) \boldsymbol{E} \\
\vdots & \vdots & \vdots & \vdots \\
J_{-2 M}(\xi) \boldsymbol{E} & J_{-2 M+1}(\xi) \boldsymbol{E} & \cdots & J_{0}(\xi) \boldsymbol{E}
\end{array}\right] \text { with } \quad \xi=\left\{\begin{array}{cc}
-\frac{\pi K \rho \kappa}{(1+\rho \kappa)} & \text { for } j=1 \\
\frac{\pi K}{(1+\rho \kappa)} & \text { for } j=2
\end{array}\right.
$$

where $\boldsymbol{E}$ is a unit matrix of size 4 .

The definitions of the matrices $\boldsymbol{A}_{1}, \boldsymbol{A}_{2}$ and $\boldsymbol{B}$ and the vector $\boldsymbol{C}$ in the boundary condition (4.8) are the following:

$$
\boldsymbol{A}_{j}=\left[\begin{array}{ccccccc}
\boldsymbol{a}_{j,-M} & \boldsymbol{a}_{j,-M}^{+} & \boldsymbol{O} & \boldsymbol{O} & \cdots & \boldsymbol{O} & \boldsymbol{O} \\
\boldsymbol{a}_{j,-M+1}^{-} & \boldsymbol{a}_{j,-M+1} & \boldsymbol{a}_{j,-M+1}^{+} & \boldsymbol{O} & \cdots & \boldsymbol{O} & \boldsymbol{O} \\
\boldsymbol{O} & \boldsymbol{a}_{j,-M+2}^{-} & \boldsymbol{a}_{j,-M+2} & \boldsymbol{a}_{j,-M+2}^{+} & \cdots & \boldsymbol{O} & \boldsymbol{O} \\
\vdots & \vdots & \vdots & \vdots & \cdots & \vdots & \vdots \\
\boldsymbol{O} & \boldsymbol{O} & \boldsymbol{O} & \boldsymbol{O} & \cdots & \boldsymbol{a}_{j, M}^{-} & \boldsymbol{a}_{j, M}
\end{array}\right]
$$

with

$$
\begin{aligned}
\boldsymbol{a}_{1, m}= & {\left[\begin{array}{cccc}
0 & 1 & 0 & 0 \\
1 & 0 & 0 & 0 \\
\alpha_{1}^{2} & 0 & 1 & 0 \\
0 & -\left(3 \alpha_{1}^{2}+i 2 m\right) & 0 & 1 \\
i \pi K & 0 & 0 & 0
\end{array}\right] \text { and } } \\
\boldsymbol{a}_{2, m}= & {\left[\begin{array}{cccc}
0 & -1 & 0 & 0 \\
-\kappa & 0 & 0 & 0 \\
-\rho \kappa \alpha_{2}^{2} & 0 & -\rho \kappa & 0 \\
0 & \rho\left(3 \alpha_{2}^{2}+i 2 m\right) & 0 & -\rho \\
0 & 0 & 0 & 0
\end{array}\right] }
\end{aligned}
$$

$\boldsymbol{a}_{1, m}^{-}$and $\boldsymbol{a}_{2, m}^{-}$have sizes of 5 -by- 4 for $m \neq 0$ and of 4 -by- 4 for $m=0$. All their elements are null except their $(4,1)$ elements. These elements are $i 2 \pi K \widetilde{W}_{1,1}^{\prime}$ for $\boldsymbol{a}_{1, m}^{-}$and $-\rho i 2 \pi K \widetilde{W_{2,1}^{\prime}}$ for $\boldsymbol{a}_{2, m}^{-}$. The matrices $\boldsymbol{a}_{1, m}^{+}$and $\boldsymbol{a}_{2, m}^{+}$are given by $\boldsymbol{a}_{1, m}^{+}=-\left(\boldsymbol{a}_{1, m}^{-}\right)^{*}$ and $\boldsymbol{a}_{2, m}^{+}=-\left(\boldsymbol{a}_{2, m}^{-}\right)^{*}$. 


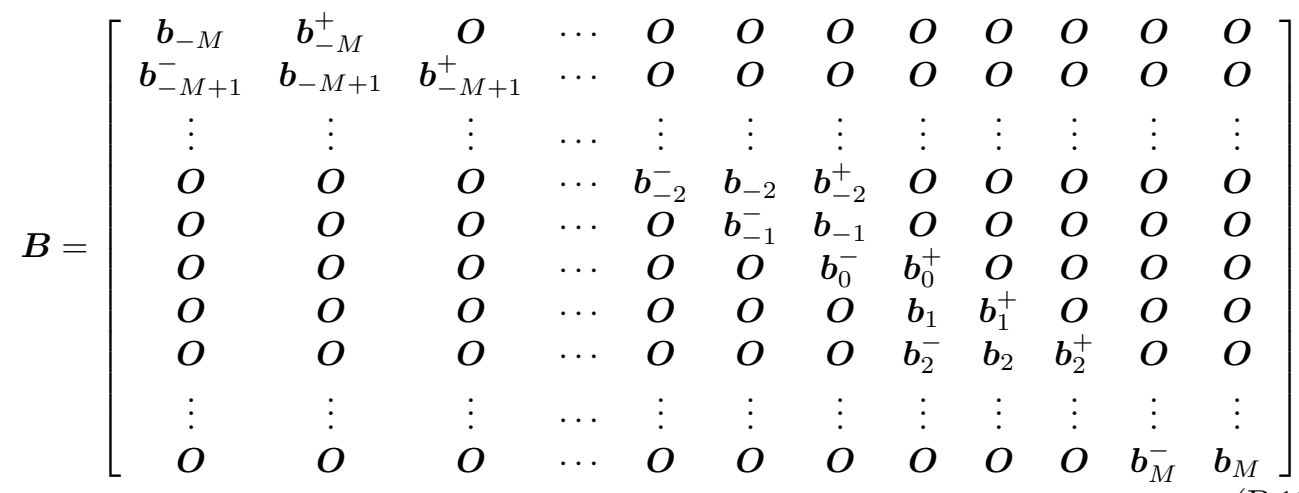

with

$$
\boldsymbol{b}_{m}=\left[\begin{array}{c}
0 \\
0 \\
0 \\
-i \frac{\pi^{2} \rho K\left(1+q^{2}\right)}{\kappa(1+\rho) \mathrm{B} \sqrt{\Omega}} \\
i m
\end{array}\right]
$$

The matrices $\boldsymbol{b}_{m}^{-}$have sizes of 5 -by- 1 for $m \neq 0$ and of 4 -by- 1 for $m=0$. Their elements are null except the first and third ones. The first element is $\left(\rho \kappa^{2}-1\right) \widetilde{W}_{1,1}^{\prime} / \rho \kappa^{2}$ and the third one is $(\kappa+1) \widetilde{W}_{1,1}^{\prime \prime} / \kappa$. The matrices $\boldsymbol{b}_{m}^{+}$are given by $\boldsymbol{b}_{m}^{+}=\left(\boldsymbol{b}_{m}^{-}\right)^{*}$.

$$
\boldsymbol{C}=\left[\begin{array}{c}
0 \\
\vdots \\
0 \\
\boldsymbol{c}_{-1} \\
\boldsymbol{c}_{0} \\
\boldsymbol{c}_{1} \\
0 \\
\vdots \\
0
\end{array}\right] \quad \text { with } \quad \boldsymbol{c}_{0}=\left[\begin{array}{c}
0 \\
0 \\
0 \\
i \frac{\pi^{2} \rho K\left(1+q^{2}\right)}{\kappa(1+\rho) \sqrt{\Omega} \mathrm{B}}
\end{array}\right], \quad \boldsymbol{c}_{1}=\left[\begin{array}{c}
-\frac{\rho \kappa^{2}-1}{\rho \kappa^{2}} \widetilde{W}_{1,1}^{\prime} \\
0 \\
-\frac{\kappa+1}{\kappa} \widetilde{W}_{1,1}^{\prime \prime} \\
0 \\
0
\end{array}\right]
$$

and $\boldsymbol{c}_{-1}=\left(\boldsymbol{c}_{1}\right)^{*}$. The first and last $5(M-1)$ elements of $\boldsymbol{C}$ are null.

\section{REFERENCES}

Beysens, D., Wunenburger, R., Chabot, C. \& Garrabos, Y. 1998 Effect of oscillatory accelerations on two phase fluids. Microgravity Sci. Technol. 11 (3), 113-118.

Greenspan, H. P. 1969 The theory of rotating fluids. London: Cambridge University Press.

Hinch, E. 1984 A note on the mechanism of the instability at the interface between two shearing fluids. J. Fluid Mech. 144, 463-465.

Hooper, A. P. \& Boyd, W. G. C. 1983 Shear-flow instability at the interface between two viscous fluids. J. Fluid Mech. 128, 507-528.

Ivanova, A. A., Kozlov, V. G. \& Evesque, P. 2001 a Interface dynamics of immiscible fluids under horizontal vobration. Fluid Dynamics 36, 362.

Ivanova, A. A., Kozlov, V. G. \& Tachkinov, S.I. $2001 b$ Interface dynamics of immiscible fluids under circularly polarized vibration (experiment). Fluid Dynamics 36, 871. 
JALIKoP, S. \& JUEL, A. 2009 Steep capillary-gravity waves in oscillatory shear-drived flows. J. Fluid Mech. 640, 131-150.

Joseph, D. D. \& Renardy, Y. 1992 Fundamentals of Two-Fluid Dynamics: Part 1: Mathematical Theory and Applications. Springer.

Kelly, R. E. 1965 The stability of an unsteady Kelvin-Helmholtz flow. J. Fluid Mech. 22, 547.

LAmb, Sir. H. 1945 Hydrodynamics, 6th edn. New York: Dover.

Lyubimov, D. V. \& Cherepanov, A. A. 1987 Development of a steady relief at the interface of fluids. Fluid. Dyn. Res. 22, 849.

Rousseaux, Germain, Yoshikawa, Harunori, Stegner, Alexandre \& Wesfreid, José EduARdo 2004 Dynamics of transient eddy above rolling-grain ripples. Phys. Fluids 16 (4), 1049-1058.

Sнyн, C. K. \& Munson, B. R. 1986 Interfacial instability of an oscillating shear layer. J. Fluid Eng. 108, 89-92.

Sleath, J. F. A. 1984 Sea bed mechanics. Wiley.

Stegner, A. \& Wesfreid, J. E. 1999 Dynamical evolution of sand ripples under water. Phys. Rev. E 60 (4), R3487-R3490.

Talib, Emma, Jalikop, Shreyas V. \& Juel, Anne 2007 The influence of viscosity on the frozen wave instability: theory and experiment. J. Fluid Mech. 584, 45-68.

Vittori, G. 1989 Non-linear viscous oscillatory flow over a small amplitude wavy wall. $J$. Hydraul. Res. 27 (2), 267-280.

Vittori, G. \& Blondeaux, P. 1990 Sand ripples under sea-waves. Part 2. Finite amplitude development. J. Fluid Mech. 218, 19-39.

Wolf, G. H. 1969 The dynamic stabilization of the Rayleigh-Taylor instability and the corresponding dynamic equilibrium. Z. Physik 227, 291-300.

Yıн, C.-S. 1967 Instability due to viscosity stratification. J. Fluid Mech. 26, 337.

Yoshikawa, H. N. \& Wesfreid, J. E. 2010 Oscillatory Kelvin-Helmholtz instability: Part 1. A viscous theory. J. Fluid Mech. (in press). 